\title{
Affective value and associative processing share a cortical substrate
}

\author{
Amitai Shenhav • Lisa Feldman Barrett • Moshe Bar
}

Published online: 23 October 2012

(C) Psychonomic Society, Inc. 2012

\begin{abstract}
The brain stores information in an associative manner so that contextually related entities are connected in memory. Such associative representations mediate the brain's ability to generate predictions about which other objects and events to expect in a given context. Likewise, the brain encodes and is able to rapidly retrieve the affective value of stimuli in our environment. That both contextual associations and affect serve as building blocks of numerous mental functions often makes interpretation of brain activation
\end{abstract}

Electronic supplementary material The online version of this article (doi:10.3758/s13415-012-0128-4) contains supplementary material, which is available to authorized users.

\section{A. Shenhav}

Department of Psychology, Harvard University,

Cambridge, MA 02138, USA

A. Shenhav $\cdot$ L. F. Barrett $\cdot$ M. Bar

Martinos Center for Biomedical Imaging

at Massachusetts General Hospital, Harvard Medical School,

Charlestown, MA 02129, USA

L. F. Barrett • M. Bar

Department of Psychiatry, Massachusetts General Hospital,

Harvard Medical School,

Charlestown, MA 02129, USA

L. F. Barrett

Department of Psychology, Northeastern University,

Boston, MA 02115, USA

M. Bar

Gonda Center for Multidisciplinary Brain Research,

Bar-Ilan University,

Ramat Gan 52900, Israel

\section{A. Shenhav $(\square)$}

Princeton Neuroscience Institute, Princeton University,

Green Hall, 3-N-12,

Princeton, NJ 08540, USA

e-mail: ashenhav@princeton.edu ambiguous. A critical brain region where such activation has often resulted in equivocal interpretation is the medial orbitofrontal cortex (mOFC), which has been implicated separately in both affective and associative processing. To characterize its role more unequivocally, we tested whether activity in the mOFC was most directly attributable to affective processing, associative processing, or a combination of both. Subjects performed an object recognition task while undergoing fMRI scans. Objects varied independently in their affective valence and in their degree of association with other objects (associativity). Analyses revealed an overlapping sensitivity whereby the left mOFC responded both to increasingly positive affective value and to stronger associativity. These two properties individually accounted for mOFC response, even after controlling for their interrelationship. The role of the mOFC is either general enough to encompass associations that link stimuli both with reinforcing outcomes and with other stimuli or abstract enough to use both valence and associativity in conjunction to inform downstream processes related to perception and action. These results may further point to a fundamental relationship between associativity and positive affect.

Keywords Valence $\cdot$ Context $\cdot$ Prefrontal cortex $\cdot$ Object recognition

\section{Introduction}

Affective and associative processes are both crucial for an individual's ability to understand and act in the world. To best anticipate how to respond to a newly presented object, the brain immediately begins the process of object recognition, while also extracting the motivational relevance (or affective value) of that object. In so doing, the brain quickly and efficiently activates relevant associations that give rise 
to focused predictions (i.e., "what other objects or contexts might go with this object?"; Bar, 2004, 2009; Chun \& Jiang, 2003; Oliva \& Torralba, 2007). The ease with which a stimulus brings to mind other related stimuli and contexts (what we will refer to as its associativity) ${ }^{1}$ is central to research into memory, prospection, imagination, and scene construction (Bar, 2004; Bar, Aminoff, Mason, \& Fenske, 2007; Barsalou, 2009; Bartlett, 1932; Bower, 2008; Eichenbaum \& Fortin, 2009; James, 1890). Similarly, the brain quickly predicts an object's affective value - in particular, its valence (i.e., "is this something pleasant/approachable or unpleasant/to-be-avoided?"; Barrett \& Bliss-Moreau, 2009; Cabanac, 2002; Damasio, 1994; Rolls, 1986; Russell, 2003). These two domains of prediction are supported by vast yet largely nonoverlapping psychological and neuroscientific literatures, despite indications that they might share some cognitive and neural mechanisms (Andrews-Hanna, Reidler, Sepulcre, Poulin, \& Buckner, 2010; Barrett \& Bar, 2009; D'Argembeau et al., 2009). One region in particular that is consistently implicated in both fields of research is the medial orbitofrontal cortex (mOFC; meta-analytic summary in Roy, Shohamy, \& Wager, 2012).

Cognitive neuroscientific research has shown that regions of the ventromedial prefrontal cortex (PFC; including the mOFC) are involved in tasks that engage associative processing, including those that do so through recall of past autobiographical experiences (e.g., Burianova \& Grady, 2007; Denkova, Botzung, Scheiber, \& Manning, 2006), imagination of possible future events (e.g., Addis, Wong, \& Schacter, 2007; Peters \& Buchel, 2010; Szpunar, Watson, $\&$ McDermott, 2007), mind-wandering (e.g., Christoff, Gordon, Smallwood, Smith, \& Schooler, 2009; Mason et al., 2007), and perception of contextually associative stimuli (objects/scenes; e.g., Bar, 2004; Bar \& Aminoff, 2003). These research areas have shown that the mOFC is more active as tasks elicit greater associative processing (reviewed in Bar et al., 2007; Buckner, Andrews-Hanna, \& Schacter, 2008; for a related meta-analytic summary, see Spreng, Mar, \& Kim, 2009).

Other areas of human neuroscience have extensively explored the mOFC's role in affective processing. This has been studied in the context of perception for both basic stimuli (e.g., tastes, smells; Grabenhorst \& Rolls, 2008; Rolls, Kringelbach, \& de Araujo, 2003) and complex stimuli (e.g., emotional faces, affect-laden scenes; Nielen et al., 2009; O'Doherty, Winston, et al., 2003), extinction of conditioned emotional responses (e.g., Kalisch et al., 2006; Milad et al., 2007), choices between different magnitudes

\footnotetext{
${ }^{1}$ We note that while affective predictions are also based on associations -namely, associations between stimuli and reinforcing outcomes-we will use the term associative to refer to stimulus-stimulus and/or stimulus-context associations exclusively (irrespective of object valence).
}

and types of rewarding and/or aversive stimuli (e.g., Kim, Shimojo, \& O'Doherty, 2006; O'Doherty, Kringelbach, Rolls, Hornak, \& Andrews, 2001), and updating of associated outcome expectations over time (e.g., Daw, O'Doherty, Dayan, Seymour, \& Dolan, 2006; Hampton, Bossaerts, \& O'Doherty, 2006; Seymour et al., 2005). Across these domains, mOFC activity typically correlates with whether and/or to what degree a stimulus is more pleasant or preferred (i.e., more positively valenced; meta-analytic summaries in Brown, Gao, Tisdelle, Eickhoff, \& Liotti, 2011; Grabenhorst \& Rolls, 2011; Kringelbach \& Berridge, 2009; Liu, Hairston, Schrier, \& Fan, 2011).

Because these areas of research traditionally take place relatively independently of one another and with different types of stimuli/paradigms, the intersection between affective and associative processes and the shared neural architecture that underlies them remain poorly understood. This is important not only because it represents a crucial gap in our understanding of how these systems and processes interact, but also because it introduces some degree of ambiguity when interpreting the results of a given study. Specifically, the centrality of the mOFC to a large body of research in these two separate fields, combined with the fact that both contextual associations and affective value appear to be processed automatically in perception and cognition (Bargh \& Chartrand, 1999; Zajonc, 1980), makes it possible that studies intending only to manipulate affect observed activation in the mOFC related to the associative nature of their stimuli but attributed it to affect and vice versa (cf. Peters, 2011). We were therefore particularly interested in elucidating whether the mOFC's presumed stimulus processing role in one of these dimensions is accounted for by the other.

To address this key issue, in the present study, we examined both affective and associative processing in the mOFC within a single experiment, using stimuli that varied parametrically along each of these dimensions. Specifically, using a single paradigm, we tested whether the same region of the mOFC would show increased activity in response to objects with greater positive affective value (higher valence), as well as to objects that are better able to elicit associations (higher associativity). One possibility is that the $\mathrm{mOFC}$ performs a processing role corresponding exclusively to either affective or associative processing. If this is the case, our experiment should confirm that mOFC activity is entirely accounted for by either valence or associativity, suggesting that it is possible to reduce one psychological domain to the other. A second possibility is that the mOFC is a hetero-functional region that separately processes information along these different dimensions or performs a more general function to which both affective and associative information contribute. In this case, the mOFC might track each object dimension independently, resulting in a purely 
additive influence of the two in BOLD signal measured within the mOFC. A third possibility is that mOFC responsiveness to valence and associativity might be dominated by an interaction between these two dimensions.

We first replicate previously separate sets of results showing BOLD activity in the mOFC tracking positive value and increasing associativity simultaneously during object perception. Importantly, we extend these findings to show that, from the perspective of mOFC activity, neither of these dimensions is reducible to the other; valence and associativity are independently and additively related to activity in the mOFC. Finally, we provide preliminary evidence suggesting that associativity-selectivity and valenceselectivity, at the voxel level, may not simply arise from separate regions within the mOFC.

\section{Method}

\section{Subjects}

Twenty-three healthy right-handed subjects with no reported history of neurological or affective disorders and normal or corrected-to-normal visual acuity and color discrimination abilities were recruited for the fMRI experiment. Three subjects were excluded for excessive missed trials $(>30 \%$ of total trials), and 1 for an incomplete session. Thus, these results will reflect analysis of 19 total subjects (12 female; age, 19-36 years; mean age, 23.8). Written informed consent was obtained prior to the start of the scanning session, in accordance with a Human Studies Protocol (\#2001P001754) approved by Massachusetts General Hospital.

\section{Image acquisition}

Images were acquired using a Siemens $3 \mathrm{~T}$ Trio Tim MR magnet and a 32-channel RF head coil. We acquired functional image volumes as $\mathrm{T} 2 *$-weighted echo-planar images (EPIs) with the following parameters: 36 interleaved slices, 2,200-ms TR, 28-ms TE, 2.5-mm thickness, 0.75-mm gap, $64 \times 64$ matrix, 200-mm FOV (resulting in an inplane voxel size of $3.125 \times 3.125 \times 2.5 \mathrm{~mm}$ ). Our fMRI sequence and slice prescription was optimized for reducing signal loss and distortion in the orbitofrontal cortex (OFC), including the use of a modified $z$-shim prepulse moment and $30^{\circ}$ tilt of our slice prescription counterclockwise of the AC/PC line along the sagittal plane (based on recommendations of Deichmann, Gottfried, Hutton, \& Turner, 2003). As a consequence of the limited slice prescription used in order to achieve optimal OFC signal, the most dorsal portions of the posterior parietal cortex were not captured in the scan volume for a majority of subjects (13 out of 19). Each subject performed three functional runs, each consisting of 149
TRs. Each run included $11 \mathrm{~s}$ of fixation at the beginning (to allow for the fMRI signal to reach steady-state), and the corresponding five EPI volumes were discarded from further analysis. Each session included the acquisition of two high-resolution T1-weighted Multi-Echo MPRAGE anatomical images (1-mm isotropic voxels), which were later averaged together.

\section{Stimulus norming}

Our analyses explored three categories of valence (negative, neutral, positive) and two categories of associativity (weak and strong). Our first stage of analysis focused on replicating previously separate findings in as broad a stimulus set as possible. For this stage, associativity analyses were therefore limited to neutrally valenced objects previously normed by Bar and Aminoff (2003). Our second stage of analysis was finer-grained and used object categories that fully crossed levels of valence and associativity, based on stimulus renorming with a large group of raters. (See also the Supplementary Discussion regarding motivation for and potential limitations of using independent ratings.)

\section{Valence}

Images of strongly and weakly associative objects were initially compiled from a set used in Bar and Aminoff (2003; available at http://barlab.mgh.harvard.edu/ ContextLocalizer.htm), as well as additional stimuli previously compiled using similar norming procedures. These neutrally valenced objects were supplemented with images of positive and negative affect-inducing objects compiled and modified primarily from the International Affective Picture System (Lang, Bradley, \& Cuthbert, 2008), as well as from online image searches. Each of the resulting 462 objects was viewed by 7 independent raters (14 raters total, each viewing half of the set), who provided valence ratings on a 7-point Likert scale ranging from very unpleasant to very pleasant (centered on neutral). The averaged ratings and their variances were used to limit this set to objects consistently rated as positive (pleasant) and negative (unpleasant). These ratings were also used to limit objects previously categorized as strongly/weakly associative to those consistently rated as neutral. After further limiting the set to semantically nonoverlapping stimuli, the resulting set of stimuli included 276 objects - 69 each of positive (valence $M \pm S D: 5.71 \pm 0.43)$, negative $(2.18 \pm 0.39)$, weakly associative neutral $(4.14 \pm 0.45)$, and strongly associative neutral $(4.18 \pm 0.45)$. This set of 276 objects was used in the first stage of analysis. To allow for the second stage of analysis, each of these objects was renormed for valence by an average of 39 new raters (range: 34-43), using the same procedure as above, and the stimulus set 
was further limited to orthogonalize these ratings against ratings of associativity collected for the same objects (as described in the following section; see also Table 1).

\section{Associativity}

The set of 276 objects described above was presented to an independent set of raters to determine the ease with which each image elicited associations with other objects. Each image was presented to an average of 39 raters (range: $35-$ 47; 116 individuals each viewed approximately one third of the total image set). Each image was presented separately, and while it remained on the screen, raters were asked to try their best to type the names of three separate objects that they associated with it. For instance, an image of a washing machine might elicit "detergent," "clothes," and "dryer" as associates. Raters' response time on this task was not limited, and they were required to produce at least one associate per image. This procedure allowed us to derive our key measures of associativity for a given image, including the number of times that an object failed to produce all three associates (i.e., the proportion of associate slots left unfilled for a given object across subjects; ease/difficulty of association) and the response time (RT) to associate production (normalized for both word length and each rater's mean typing rate; speed of association). These two measures - ease and speed of association-were significantly correlated across objects, $r(276)=$ $.53, p<.001$, and so were normalized and averaged together to form a single associativity index (with higher values representing objects that produced associations more easily and faster - thus, strongly associative). If, in the example above, the washing machine consistently produced only one or two associates and/or those associates took a long time to be generated, its associativity index would be low, and we would describe it as weakly associative. If the reverse were true (i.e., all three associates were consistently given and relatively quickly), it would be considered strongly associative. While some raters participated in both valence and associativity norming, a given rater viewed each object only once, providing either a valence or an associativity rating for that object.

For both analysis stages, these variables are discretized into categorical rather than continuous variables. The motivation for this was twofold. First, this is the approach used by a number of studies whose findings we were most closely attempting to replicate, for both associativity (e.g., Bar \& Aminoff, 2003) and valence (e.g., Nielen et al., 2009; Ritchey et al., 2011; Sass et al., 2011). Second, because valence and associativity turned out to be intercorrelated (see the Results section), the second set of analyses tested for fully independent effects of the two object dimension on mOFC activity by forming stimulus categories matched along the potentially confounding dimension. This approach to orthogonalizing our variables of interest, which we determined to have less potential of being overly conservative than alternatives, partially guided the decision to use categorical variables for all of our main analyses (see also the Supplementary Analysis).

\section{Task design}

The paradigm employed a rapid event-related design. Target images were color photographs $(256 \times 256$ pixels $)$ of objects in isolation presented on a white background. Each target image was presented briefly $(150 \mathrm{~ms})$ and was immediately followed by a colorful mask presented for $100 \mathrm{~ms}$ (see Fig. 1). This was done in order to encourage the subject to bring to mind the object and its internal associations (affective and contextual) and minimize the degree to which the subject was modulating attention toward the image of the object in front of them and the individual features thereof, as well as to encourage the subject to answer relatively automatically, rather than to consciously deliberate over potential responses. A red fixation cross then appeared signaling the start of the response period and turned

Table 1 Means (standard deviations) for valence ratings (top) and associativity index (middle) after controlling for associativity (across valence categories) and valence (across associativity categories)

\begin{tabular}{llccc}
\hline & & Negative & Neutral & Positive \\
\hline Valence (standardized) & Weak & $-0.97(0.21)$ & $0.06(0.18)$ & $0.82(0.29)$ \\
& Strong & $-0.95(0.26)$ & $0.08(0.20)$ & $0.82(0.22)$ \\
Associativity (standardized) & Weak & $-0.194(0.160)$ & $-0.194(0.185)$ & $-0.192(0.183)$ \\
& Strong & $0.341(0.162)$ & $0.340(0.197)$ & $3.46(0.65)$ \\
Commonality (1-5 scale) & Weak & $2.02(0.61)$ & $3.41(0.76)$ & $3.44(0.54)$ \\
& Strong & $2.05(0.54)$ & $(0.55)$ \\
\hline
\end{tabular}

Note. Each cell represents an average over 21 objects. This object resorting (used in the second analysis stage) was performed only on the basis of independent ratings of valence and associativity, but mixed-effects analyses of response time from subjects performing the task in the scanner reveal no significant differences across or between categories (associativity, $F(1,18)=0.44 ;$ valence, $F(2,36)=1.91$; interaction, $F(2,36)=0.76$; all $p \mathrm{~s}>.15$ ). Commonality ratings given by these subjects (bottom; with between-subjects standard deviation) did not differ significantly by associativity, but negatively valenced objects were again rated as less common (follow-up parametric analyses show that effects of valence and associativity remain after removing variance accounted for by these ratings; see the Supplementary Materials). 


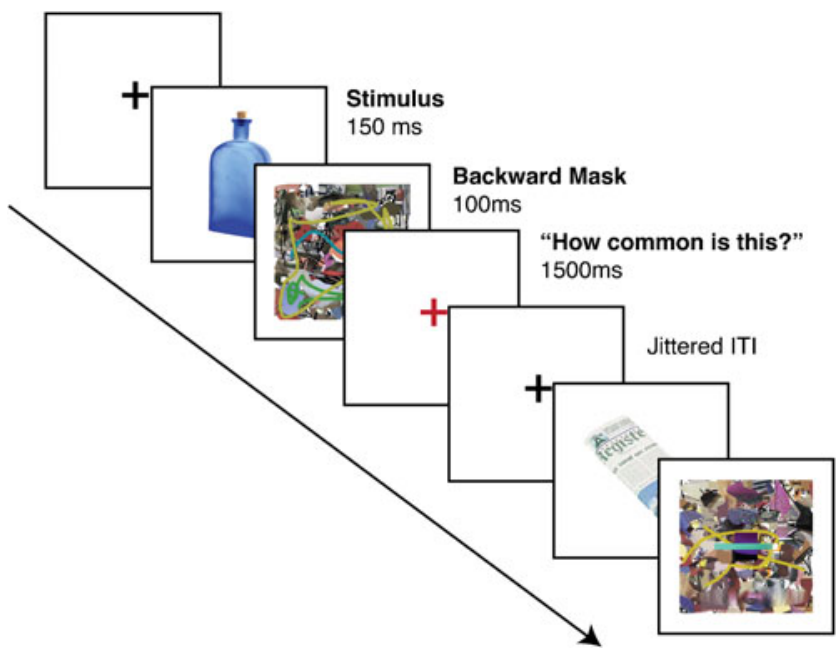

Fig. 1 Task timeline. Subjects viewed objects presented briefly and in isolation $(150 \mathrm{~ms})$, followed by a colorful backward mask (100 ms), and then rated how common the object was (1.5-s response period). In between trials, subjects viewed a black fixation cross for variable durations

black after 1,500 ms, signaling the end of the response period. The black fixation cross remained on the screen for the duration of the intertrial interval (ITI), which ranged from 200 to 9,250 ms (to allow a jittered ITI in multiples of TR length and jittered stimulus presentation from the start of each TR). The fMRI session consisted of 276 unique trials, pseudorandomly ordered across three functional runs, in addition to 28 practice trials (practice trials presented objects not included in the main task). Each target image was presented only once.

Subjects were instructed to rate how common each object was on a 5-point scale ranging from not at all common to extremely common. This task was chosen in order to require some high level of object recognition without focusing attention explicitly on either affective or associative qualities of the object, an assumption we later verify by comparing ratings of commonality across image categories. Responses were provided on a five-button MR-compatible response box. The order of the 5-point scale was counterbalanced across subjects to prevent confounds between rating and motor mapping, and subjects practiced the appropriate mapping to proficiency before the practice trials began. Stimulus presentation and response collection was performed using Psychtoolbox (http:// www.psychtoolbox.org; Brainard, 1997) running on MATLAB (http://www.mathworks.com), controlled by a MacBook Pro laptop with a monitor resolution of $1,024 \times$ 768 and a refresh rate of $60 \mathrm{~Hz}$.

\section{fMRI analysis}

Structural and functional imaging analyses were performed using the Freesurfer and FS-FAST analysis tools and processing stream developed at the Martinos Center for Biomedical
Imaging (http://surfer.nmr.mgh.harvard.edu). Data from individual fMRI runs were first motion corrected using the AFNI motion correction algorithm (http://afni. nimh.nih.gov/afni/), in which all images were aligned to the first image of the first functional run. The data were then spatially smoothed using a Gaussian fullwidth at half-max (FWHM) of $6 \mathrm{~mm}$. The first five volumes were removed from each fMRI run to allow for signal stabilization. The intensities for all runs were globally rescaled such that the in-brain mean intensity was 1,000 . Signal intensity for each condition was then computed and averaged throughout all the runs. Each subject's fMRI volumes were also co-registered to their own high-resolution structural volume, and a semiautomated procedure was used to segment this structural data into gray/white matter components and to extract the outer cortical surface for each hemisphere as a topologically preserved spherical representation (software and documentation is available at http://www.nmr.mgh.harvard.edu/ freesurfer).

For the first analysis stage, all events were modeled according to the four object categories, and for the second analysis stage, an additional condition modeled any stimuli excluded from the six more conservatively defined categories. Analyses reported include any trials where subjects failed to respond in time (which were infrequent and not influenced by object category; see the Behavioral Results section), but all results remain unchanged if these are modeled as a separate condition (see also the Supplementary Analysis). The estimated hemodynamic response was defined by a gamma function of 2.25 -s hemodynamic delay and 1.25-s dispersion. Data were then tested for statistical significance for each individual (first level), and contrast maps were constructed comparing the BOLD estimates for each condition. While the a priori focus of our analyses was on a specific set of regions of interest (ROIs; described below), to allow comparison with previous findings and test for overlap between key contrasts, whole-brain random effects analyses were performed at the group (second) level with an omnibus $F$-test and individual $t$-tests over contrast maps generated at the first level. In order to reduce the influence of noise at the first level, these random effects analyses weighted first-level contrast maps by the inverse of their variance (Thirion et al., 2007). Whole-brain statistical maps were corrected for multiple comparisons using a cluster significance threshold of $p<.05$ (corrected), with a cluster-defining (voxelwise) threshold of $p<.01$. Since primary analyses are restricted to a priori ROIs, we chose a standard but relatively liberal cluster-defining threshold in order to describe the full extent of our network of activations. However, because cluster correction was performed in volumetric space, activations shown on the cortical surface (Fig. 3, left) are not cluster corrected but, instead, set at a 
more conservative voxelwise threshold of $p<.001$. All whole-brain analyses were performed for confirmatory rather than exploratory reasons (i.e., ROI selection was based strictly on anatomy, rather than functional activation). In order to visualize overlap between individual contrasts, a conjunction was performed over these corrected maps using the minimum statistic (Nichols, Brett, Andersson, Wager, \& Poline, 2005). While ROI analyses were performed on cortical surfaces in each subject's native space, group-level analyses were performed after transforming each subject's data into normalized (Talairach) space.

\section{ROI analysis}

Given a priori predictions about the involvement of the two regions in our task, we used Freesurfer's automated segmentation and parcellation algorithms (Desikan et al., 2006; Fischl et al., 2002; Fischl et al., 2004) to define anatomical ROIs within the left and right mOFC on the basis of each subject's individual anatomy (see Fig. 3b for an example). The parcellation algorithms used gyral and sulcal landmarks from each individual's surface anatomy to define an ROI on each hemisphere that extended rostrally/caudally according to the boundaries of the medial orbital gyrus, was bounded on the orbital surface by the midpoint of the olfactory sulcus, and was bounded on the medial surface by the inferior boundaries of cingulate and superior frontal gyri (Desikan et al., 2006). Averaged beta weights were extracted from this ROI for each of our conditions and then converted to percent signal change values. In order to determine whether BOLD activity in this region tracked one or both of our parameters of interest (or their interaction), these values were then entered into a mixed-effects valence $x$ associativity ANOVA model, with subject as a random effect. We used a two-tailed $p$-value less than $.025(p<$ .05 , corrected for the left- and right-hemisphere a priori ROIs) as our significance threshold in these ROI analyses, and we note the cases in which this correction was applied when reporting the results of the ROI analyses.

\section{Voxel overlap analysis}

In order to perform a more conservative test against the possibility that valence and associativity sensitivity revealed in mOFC voxels was actually occurring in segregated sets of voxels within our anatomically defined ROI, we extracted averaged percent BOLD signal change estimates for each valence condition from the 20 most associativity-selective voxels in the left mOFC. Selectivity was assessed on a within-subjects basis by rank-ordering voxels (without consideration for spatial contiguity) on the basis of $p$-values from the omnibus $F$-test for a voxel-wise general linear model (GLM) that included only category labels for strong and weak associativity. We then tested for a significant effect of valence category in these associativity-selective voxels, using a mixed-effects ANOVA. We also performed the reverse analysis, testing for associativity selectivity in the most valence-selective voxels. To test for robustness, this analysis was repeated for samples of between 1 and 100 peak voxels.

\section{Results}

Subjects performed a simple task requiring the recognition of visually presented objects while undergoing fMRI scans (Fig. 1). Images of isolated objects were presented briefly $(150 \mathrm{~ms})$, and subjects were asked to judge how common the object was. All objects were independently rated for hedonic valence and associativity, and planned ROI analyses tested for changes in mOFC BOLD signal across levels of valence and associativity. Our first stage of analysis focuses on four object categories (negative, neutral-weak, neutral-strong, positive; for examples, see Fig. 2a), defined across the full set of stimuli viewed in the scanner. Our second stage narrows this stimulus set to allow for fully crossed and orthogonalized analyses of all six possible categories (negative-weak, negative-strong, neutral-weak, neutral-strong, positive-weak, positive-strong; Fig. 4a).

\section{Behavioral results}

Subject ratings and RTs were entered into separate mixedeffect ANOVAs, with subject as a random effect. On the basis of the four initial categories, we found a significant overall main effect of condition on ratings of commonality, $F(3,54)=101.2, p<.001$, such that negative objects were consistently rated less common, on average, than objects in the other three conditions (see the Supplementary Materials for secondary analyses controlling for this). There was no significant difference between commonality ratings for the remaining three conditions on the basis of post hoc contrasts, $F(3,100)=0.92, p>.40$. There was no significant main effect of condition on RT, $F(3,54)=2.0, p>.10$, suggesting no clear difference in task difficulty across the four conditions. The lack of commonality differences (with the exception of negative objects) and lack of RT differences remained true after trials were later resorted for the second analysis stage (see Table 1 legend). Subjects missed relatively few trials (median $=2.2 \%$, mean $\pm S D=5.7 \% \pm$ $6.9 \%$ ), with no effect of condition, $F(3,54)<0.10$.

An additional finding emerged related to the independent ratings of our two object dimensions of interest. We found that an object's valence and degree of associativity were moderately correlated, $r(276)=.31, p<.0001$ (see Supplementary Fig. 1), such that objects that were rated as 
Fig. 2 Analysis of mOFC reactivity to affectively valenced categories, relative to neutral objects of varying associativity. a Examples of stimuli from each of the four object categories. b Individually defined left mOFC anatomical ROI shown on a single subject's inflated surface. c Group ROI analyses show average BOLD response to each of these four categories in the left mOFC. Error bars reflect between-subjects standard errors of the means

a

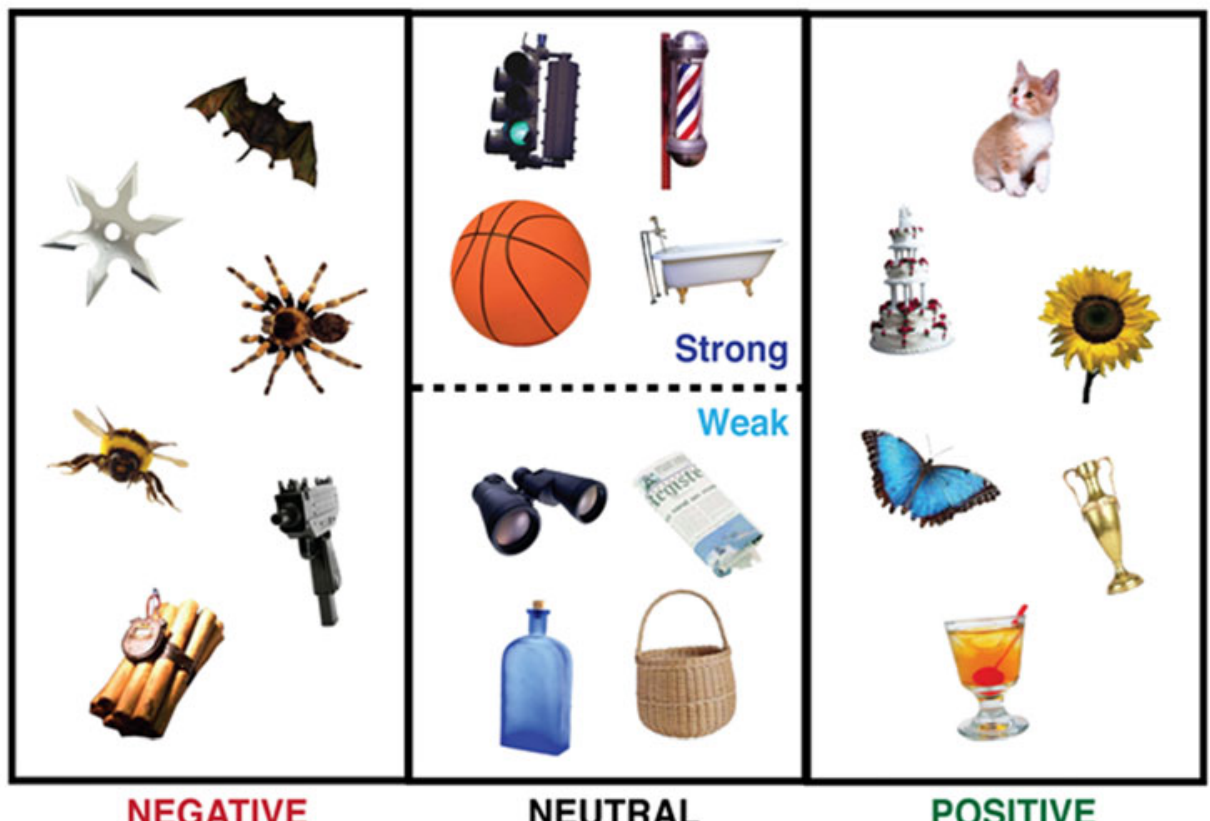

b

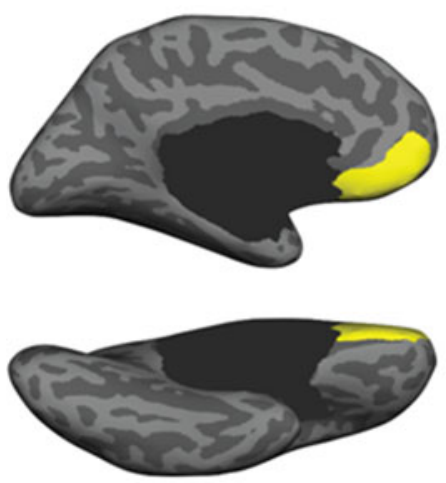

C

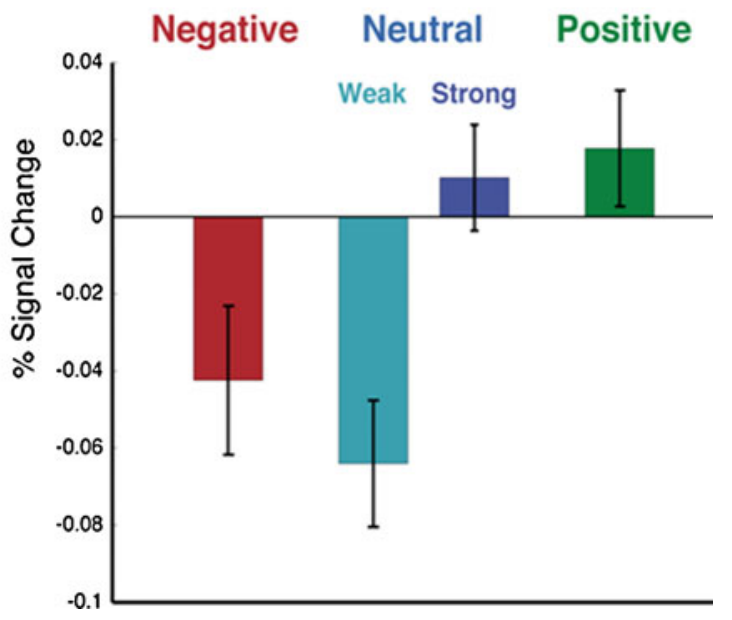

more positive tended to be more strongly associative. While this relationship is interesting in its own right and further motivates the need for disambiguation of previous mOFC findings, we also control for this as a potential confound in our second set of analyses.

Imaging results: replication of previously separate affective and associative findings

Our analyses focused on left and right mOFC a priori ROIs using surface anatomic landmarks in each subject's native space (see the Method section and the example in Fig. 2b). Since we had no clear a priori hypothesis about one hemisphere or another, our ROI analyses were Bonferronicorrected for the dual a priori hypotheses. We extracted estimates of BOLD signal from these mOFC ROIs for each of the four broader stimulus categories and found a significant main effect of condition [Fig. 2c; left, $F(3,54)=$ 19.3 ; right, $F(3,54)=8.52 ; p \mathrm{~s}<.001$, corrected]. A series of planned post hoc contrasts confirmed that both associativity and affective value engaged the mOFC. Directly replicating previous studies of associativity (Aminoff, Schacter, \& Bar, 2008; Bar \& Aminoff, 2003), we found that neutral objects with strong associations significantly increased BOLD activity in the $\mathrm{mOFC}$, when compared with weakly associative objects [left, $F(1,54)=33.3$; right, $F(1,54)=15.4 ; p \mathrm{~s}<$ .001 , corrected]. Mirroring findings for a number of different kinds of affectively valenced stimuli (Brown et al., 2011; Chib, Rangel, Shimojo, \& O'Doherty, 2009; Lebreton, Jorge, Michel, Thirion, \& Pessiglione, 2009; Nielen et al., 2009; O'Doherty, Critchley, Deichmann, \& Dolan, 2003; Sass et al., 2011), BOLD activity in the mOFC also increased significantly for positive objects, as compared with the neutrally valenced objects (i.e., neutral- 
strong and neutral-weak combined) and with the negative objects [left, $F(3,72)=8.2, p<.001$, corrected; right, $F(3,72)=3.4, p<.05$, corrected; Fig. 2c].

While our analyses focus explicitly on individually defined ROIs, results of a confirmatory group whole-brain analysis (Fig. 3, Supplementary Table 1) show that activations in the mOFC appear prominently in each of the three whole-brain corrected contrasts of interest. These analyses also reveal expected patterns of coactivation for associativity in retrosplenial and parahippocampal cortices, which have been observed across all previous studies of associativity (Bar et al., 2007) and many studies of episodic and autobiographical memory more generally (Buckner et al., 2008; Roy et al., 2012). And regions coactivated with the $\mathrm{mOFC}$ in valence contrasts, including the dorsal $\mathrm{mPFC}$ and amygdala, are also commonly coactivated with this region across studies of affective processing (Kober et al., 2008; Roy et al., 2012). A conjunction of these contrasts confirmed that the greatest degree of spatial overlap fell within the mOFC and, in particular, the left mOFC (Fig. 3e). One immediate implication of these results is that the differential activation in the mOFC to strongly versus weakly associative objects reported in previous research is robust to more careful control for valence. However, this still leaves unanswered the question of whether the effect of valence on the mOFC is mediated by associativity. We turn to this question next in our second analysis stage. Because our investigation was primarily concerned not with the function of all of the mOFC but, specifically, with those regions related to both affect and associations, the remaining analyses focus on the left mOFC, where selectivity was substantially stronger for both dimensions [as discussed above and further confirmed by a significant ROI $\times$ condition interaction: $F(3,54)=10.13, p<.0001]$. Bonferroni correction continued to be applied to adjust for our two a priori hypotheses.

Imaging results: relationship between affective and associative processing in the $\mathrm{mOFC}$

Subsequent analyses revealed that the effects of valence and associativity on mOFC activity are independent of and additive with one another, indicating that the mOFC's role in affective processing is not attributable to associativity or vice versa. To confirm that mOFC activity was independently influenced by both object properties, this more conservative second stage of analysis focused on a subset of objects that were classified into one of six possible combinations of valence and associativity level (negative-weak, etc.). These objects were also selected so that categories at the same level of associativity were matched for valence and vice versa, to address any potential confounds between the two (see Table 1 and examples in Fig. 4a). We were then able to run a 3 a
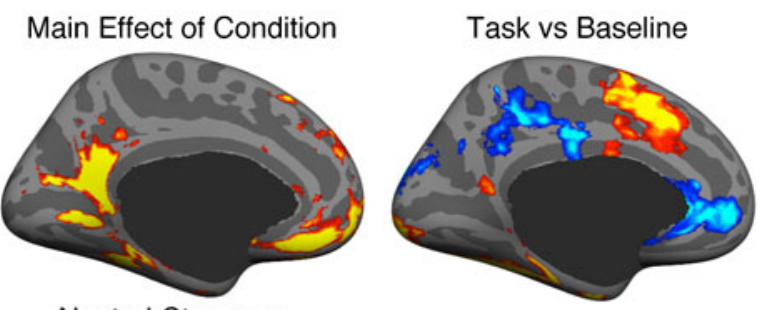

b
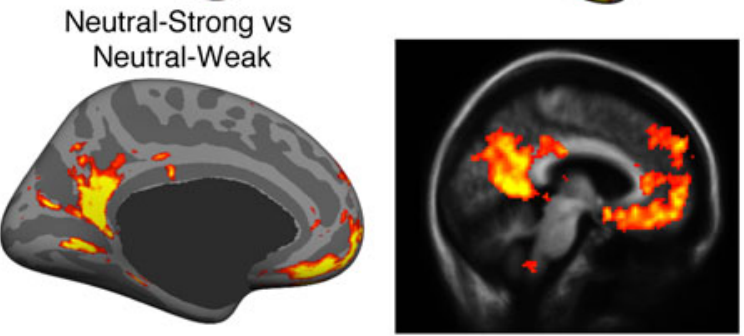

C
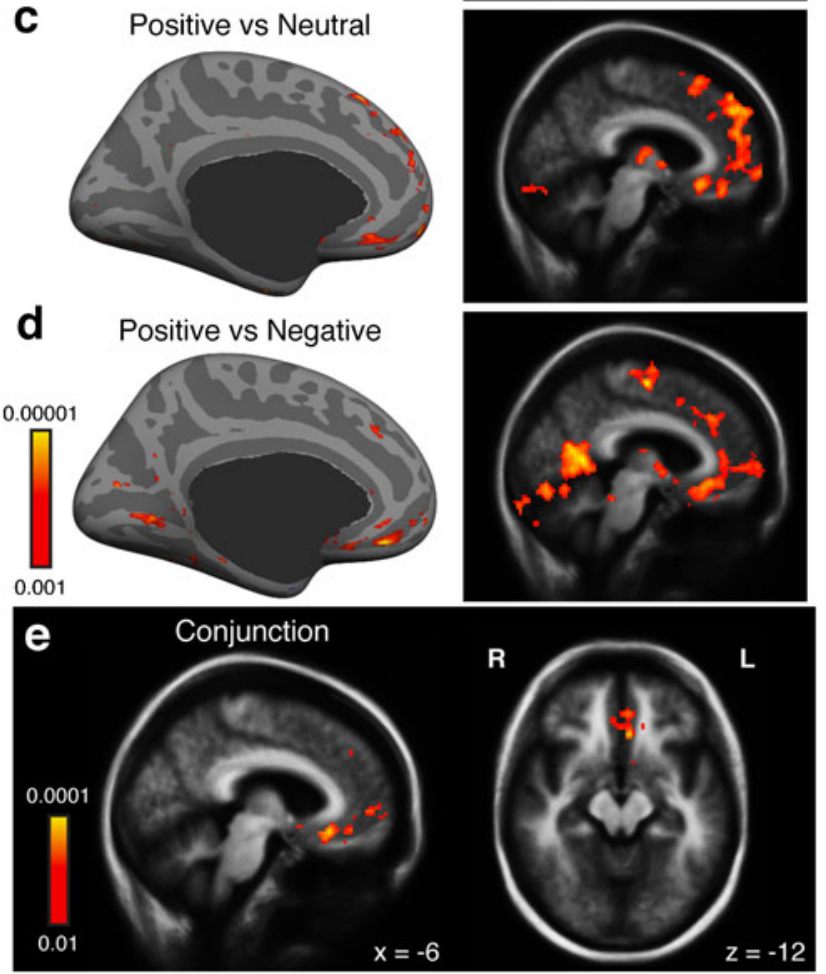

Fig. 3 a Whole-brain analyses projected onto the inflated cortical surface display the main effect of condition (left; significance values based on $F$-test for group repeated measures ANOVA) and the simple effect contrast ( $t$-test) for the average of all conditions, relative to baseline (right). b-d Whole-brain contrasts ( $t$-tests) display relative BOLD activation when viewing strongly versus weakly associative objects (neutrally valenced) and objects with positive affective value (relative to neutral and negative) (left: surface-based maps shown at uncorrected voxelwise threshold of $p<.001$; right: same activations shown on the brain volume in Talairach space at voxelwise $p<.01$, extent-thresholded to achieve a whole-brain cluster-corrected threshold of $p<.05$ ). e A conjunction of these three (corrected) contrasts reveals a role for the left $\mathrm{mOFC}$ at the intersection of affective and associative processing. Whole-brain maps provided for completeness, but analyses of interest were performed in a priori ROIs

(valence: positive, negative, neutral) $\times 2$ (associativity: strong vs. weak) ANOVA for BOLD signal in the left mOFC. We 
Fig. 4 Analysis of mOFC reactivity to fully crossed set of objects of increasing valence (negative, neutral, positive) and different associativity levels (weak vs. strong). a Examples of stimuli from each of six object categories. b Group ROI analyses in the left mOFC reveal main effects for both valence and associativity. Error bars reflect between-subjects standard errors of the means a

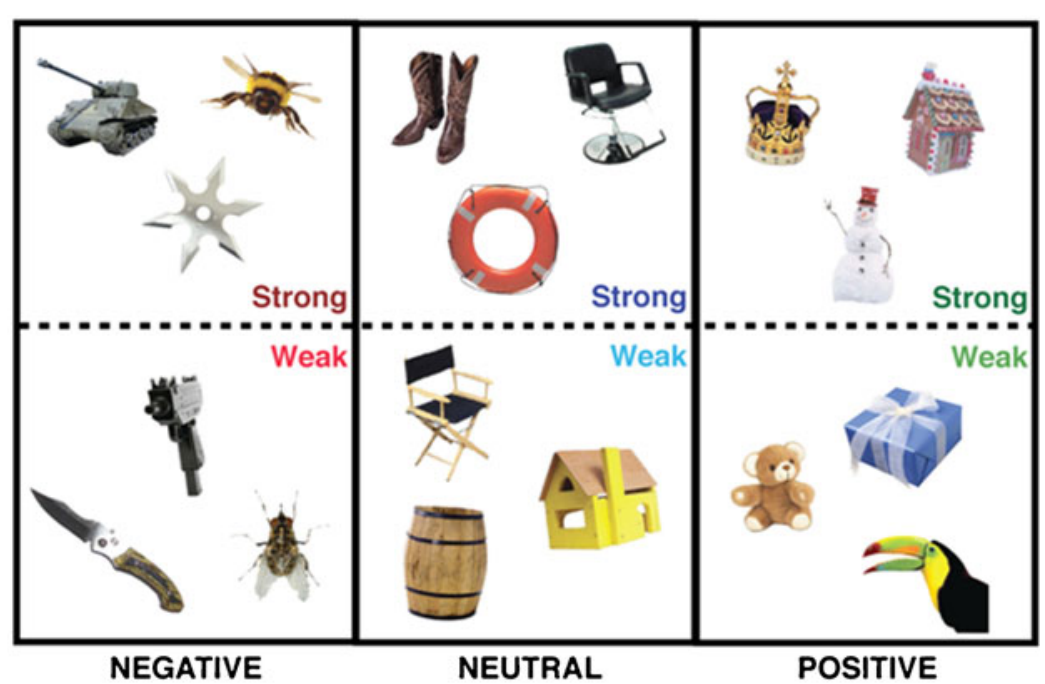

b

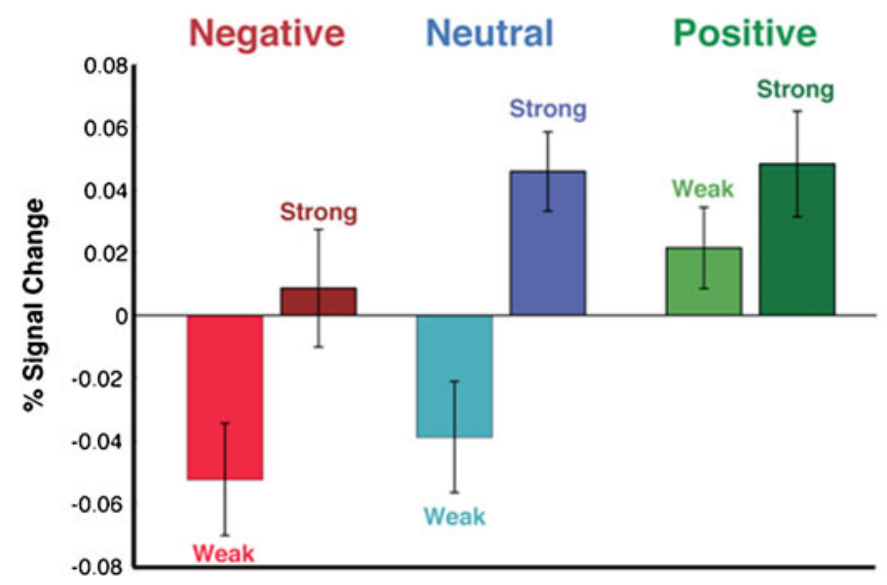

found that left mOFC BOLD activity revealed main effects of valence $[F(2,36)=4.88, p<.05$, corrected] and associativity $[F(1,18)=25.7, p<.0005$, corrected; valence $\times$ associativity: $F(2,36)=2.70, p>.15$, corrected], indicating that both properties contributed to independent increases in $\mathrm{mOFC}$ activity (Fig. 4b).

The results thus far suggest that activity in the mOFC independently increases with positive affective value and increased associativity. One possibility that remains is that these results arise from two segregated but partially overlapping regions within the left $\mathrm{mOFC}$, one selective only for valence and the other selective only for associativity. This hypothesis cannot be disconfirmed by comparing peak coordinates or exploring activation overlap at differing thresholds, because it is always possible that voxels most selective for one parameter are still significantly selective for the other parameter but that their significance fails to meet the given threshold. In order to provide evidence to militate against the possibility that our independent effects of affect and associativity originate from segregated regions within the mOFC, we instead identified the 20 voxels within the
mOFC that were most selective for associativity (on a within-subjects basis) and extracted from each subject the average percent BOLD signal change for each of the valence conditions. A mixed-effects ANOVA (treating subject as a random effect) revealed a significant effect of valence within these voxels, $F(2,36)=4.98, p<.02$. We then did the same for BOLD estimates of strongly versus weakly associative objects within voxels most selective for valence and again found a marginally significant effect for associativity, $F(1,18)=4.16, p<.06$ (Supplementary Fig. 2a). ${ }^{2}$ To ensure that neither set of estimates benefited from error variance accounted for by the other parameter, voxel selection and beta estimate extraction were derived from statistically independent GLMs over the same data set (one GLM accounting only for levels of associativity and the other only levels of valence). These findings provide preliminary support for the hypothesis that overlapping populations of

\footnotetext{
${ }^{2}$ These same general results also hold when varying the number of voxels sampled between a single peak voxel and 100 peak voxels (Supplementary Fig. 2b).
} 
voxels within the mOFC were independently responsive to both an object's affective value and the strength of its associativity with other objects/contexts.

\section{Discussion}

The mOFC has been implicated in research on both affective and associative processes, raising the important question of whether one set of proposed functions better accounts for the other. In the present study, we measured mOFC BOLD activity while subjects viewed objects that were varied orthogonally in affective valence and associativity. Replicating findings from separate literatures, we demonstrated that activity in the same region of the left mOFC was proportional to an object's valence (specifically, whether it was positively valenced or not; Brown et al., 2011; Grabenhorst \& Rolls, 2011; Kringelbach \& Berridge, 2009) and to its associative strength (Aminoff et al., 2008; Bar \& Aminoff, 2003). Crucially, we found that activation in this $\mathrm{mOFC}$ region was not reducible to either valence or associativity, even after controlling for interdependencies between the two dimensions. Both properties independently and additively modulated left mOFC activity, despite the fact that neither was integral to the task at hand. Moreover, sensitivity to an object's affective value and its associative strength seemed to arise from overlapping rather than separate populations of voxels within the anatomically defined $\mathrm{mOFC}^{3}$

In terms of both structure and connectivity, it is not surprising that the mOFC seems important for both contextually associative and affective predictions. The mOFC's proposed role in object recognition is supported by its strong connectivity with magnocellular visual processing regions along the dorsal stream (Barbas, 2007a, 2007b; Carmichael $\&$ Price, 1995b); the mOFC also sends projections back to the ventral visual stream, potentially influencing early stages of object processing in the inferotemporal cortex (Kveraga, Ghuman, \& Bar, 2007; Kveraga et al., 2011; Summerfield et

\footnotetext{
${ }^{3}$ As with any neuroimaging study, our results cannot speak to whether such functional segregation exists at the neuronal level. Previous electrophysiological findings suggest that intermixed neurons in the $\mathrm{OFC}$ and $\mathrm{mPFC}$ can code for different stimulus properties (e.g., identity, value; Padoa-Schioppa \& Assad, 2006) and other decision variables (e.g., effort required, probability of outcome; Kennerley, Dahmubed, Lara, \& Wallis, 2009), distinctions that can be lost at the level of fMRI. Therefore, while we think it interesting to note that we did not find clear evidence for functional segregation in the mOFC based on the level of resolution at which neuroimaging studies are performed, we are cautious not to interpret the results as evidence of shared function of the individual neurons in this region. With this in mind, our discussion of these findings is still consistent with previous accounts of overarching regional function, including those in the research mentioned above where different sensitivities of individual neuronal populations were found (Padoa-Schioppa \& Cai, 2011; Wallis \& Kennerley, 2011).
}

al., 2006). The mOFC has also been described as a center of integration for information regarding affective significance or value, and it sends and receives projections from regions involved in processing and regulating the autonomic physiology associated with motivated states, including the amygdala, hypothalamus, cingulate cortex, and brainstem, as well as indirect projections from the ventral striatum (Carmichael \& Price, 1995a; Öngür \& Price, 2000). Furthermore, because the mOFC receives afferent projections from all sensory modalities (largely via its dense connections with lateral aspects of the OFC), it is optimally situated for linking representations to inform judgments (Grabenhorst \& Rolls, 2011; Murray \& Wise, 2010; Wallis, 2007).

Taken together, previous research suggests that the mOFC engages in predictive processing across both cognitive and affective domains by allowing for associations between a given stimulus and both its higher-order sensory relations (i.e., other stimuli/contexts) and affective outcomes (i.e., valence) to be rapidly computed and used for the proactive generation of the corresponding predictions. One possible interpretation of the present results is, therefore, that the mOFC plays different roles for a number of independent psychological functions, or that its role is fundamentally associative but that these associations take on a number of different forms. This model gains support from the fact that the diverse afferents to the mOFC described above make it an ideal information-processing hub for independently collecting and processing information related to stimulus valence (e.g., from the lateral $\mathrm{OFC}$ and the amygdala) and to associated stimuli and contexts in memory (e.g., from the hippocampus and parahippocampal cortex). Under this interpretation, our results would be a natural consequence of this region's putative role in separately maintaining and/or transferring information related to stimulus associations, defined broadly to include associations between stimuli and other stimuli/contexts and associations between stimuli and outcome values.

In contrast, another equally plausible interpretation is that the convergence of pleasantness and associativity within the mOFC suggests that this brain area subserves a more unified purpose to which both of those psychological properties relate. The affective and reinforcement literature has suggested that such a unitary function is generating positive (and/or negative; Damasio, 1994, 1996) affective states and/ or encoding positive affective value in a stimulus (Kringelbach \& Berridge, 2009; Lebreton et al., 2009; Rolls, 1986). Building in part on psychological mechanisms previously theorized to underlie emotion (Barrett, 2006; Cabanac, 2002; Damasio, 1994; Rolls, 1986; Russell, 2003; Weiskrantz, 1968), recent research into decision making and reinforcement learning has suggested that the kind of affective value encoded by the mOFC represents the expected value of options under consideration and can be 
more appropriately considered as an abstract "currency" for guiding decisions (Haber \& Knutson, 2010; Kable \& Glimcher, 2009; Rangel \& Hare, 2010; Wallis, 2007). From this perspective, our findings would raise the important question of whether determining an object's overall expected value may include/require consideration of both its affective value and its "associative value" (i.e., the ease or multiplicity of association between it and other objects/contexts in memory). This estimate of associativity can aid the expected value computation in a number of possible ways, including as a proxy measure of possible states and physical actions that must be evaluated in relation to this stimulus (Rangel, Camerer, \& Montague, 2008). From the perspective of the processes of recall and prospection, a stimulus may also gain some currency for downstream information processing if it is better able to link up stimuli in memory or allow fluid production of future plans through its promiscuous binding to other stimuli (i.e., a type of informational processing fluency; cf. Kurth-Nelson, Bickel, \& Redish, 2012; Murray \& Wise, 2010). In other words, a stimulus might be encoded as more valuable merely because it elicits a large number of associations (Bar, Shenhav, \& Devaney, 2012). For the same reason, mOFC activity may increase with the meaningfulness (and identifiability) of an object (Chaumon, Kveraga, Barrett, \& Bar, 2012) because of the associative activation that a meaningful object elicits. While the exact kind of value evinced by associativity is still a matter of speculation, if it turns out to be the case that object associativity is another kind of value being encoded in the mOFC, one would indeed expect to see the independent encoding for valence and associativity found in our data.

Our data and the interpretations offered above are also broadly consistent with a recent review by Roy et al. (2012). In integrating across research on memory and affect, the authors proposed that the overarching function of this region (specifically, the ventromedial PFC) may be described as determining a stimulus' "affective meaning," combining both conceptual and affective inputs toward this goal (e.g., Kumaran, Summerfield, Hassabis, \& Maguire, 2009). Under this view, the mOFC may integrate value representations with the actual stimuli/contexts associated with an object, consistent with the mOFC's proposed role in representing specific states and stimulus contingencies relevant to learned reward values (i.e., "model-based" learning; Bornstein, Nylen, \& Steele, 2011; Schoenbaum, Takahashi, Liu, \& Mcdannald, 2011). The result would be increased mOFC activity for increasing numbers of readily available stimuli/contexts (i.e., stronger associativity). In this respect, future studies should consider whether functional connectivity between $\mathrm{mOFC}$ and regions coactivated by increasing associativity (e.g., parahippocampal and retrosplenial cortices) predicts the degree to which choices made in the scanner reflect model-based versus model-free learning and inference (Daw et al., 2011). It would also be interesting to see whether the reverse is true for mOFC connectivity with regions like the amygdala.

It further remains to be determined how value-related encoding in the mOFC influences cognitive processing in general and the process of object association in particular. Our finding that stimulus pleasantness and associativity are correlated suggests that one possible outcome of mOFC valuation might be to modulate the extent of associative processing for a given stimulus (Bar, 2009). In this view, positive affective states are linked with disinhibited association (i.e., a greater readiness to form or activate associations between stimuli), and negative affective states are directly related to the inhibition of stimulus-stimulus associations. While the associations attached to a stimulus are more likely to be stored in the medial temporal cortex and/or sensory cortices, according to this model mOFC could exert inhibitory control over how much associative activation is afforded downstream (Bar, 2009). In this kind of model, the mOFC would be involved in overseeing the scope of associative processing such that positively valenced signals would trigger a broadening of this scope and signals indicating negative valence in an object would result in narrowing this scope through inhibition of associative processing by the mOFC (cf. Sass et al., 2011), similar to what is seen in the narrowing of attentional focus for negatively valenced stimuli (Baddeley, 1972; Gasper \& Clore, 2002). While still largely hypothetical, this model gains support from recent findings in rhesus macaques showing that the nearby perigenual anterior cingulate cortex (BA 32) projects to regions of the parahippocampal cortex in ways that would allow it to exert excitatory and inhibitory control over the local information-processing circuitry (Bunce \& Barbas, 2011). Moreover, previous behavioral studies have identified a link between positive mood states and the generation of broader associations (Brunye et al., 2012; Clore \& Huntsinger, 2007; Fredrickson, 2004; Isen, Johnson, Mertz, \& Robinson, 1985; Mason \& Bar, 2011).

Our study carries broad implications for research into affective as well as associative processing. In particular, future studies focused on mOFC function should ideally take both stimulus properties into consideration at their design and interpretation stages. While our results suggest that the processing of associativity and valence can vary independently of one another in the mOFC, we also find that valence and associativity are correlated and activate overlapping regions in the mOFC. Therefore, mOFC activity observed while varying stimuli along only one of these dimensions may be partially or even largely, although not necessarily entirely, attributable to the other. More generally, the nature of the relationship between ratings along these two dimensions (at the psychological level) remains a topic of great importance. It may be the case either that positively valenced objects facilitate associative activation and the 
generation of predictions or that the greater availability of associates for an object makes it perceived more positively or less negatively, in line with the proposal that broader associative activation is linked with better mood (Bar, 2009). Gaining a deeper understanding of how we encode and react to these two stimulus dimensions, and their interaction, will lend importantly to our broader understanding of a more fundamental relationship between factors that have been traditionally dichotomized (and provided a perhaps false appearance of independence) into the domains of cognition and affect.

Author Note We are grateful to J. Boshyan, M. Rosen, and K. Shepherd for assistance in data collection, T. Benner for technical guidance, E. Aminoff for assistance in stimulus collection, and S. Gagnon for assistance with analysis and helpful comments on the manuscript. This work was supported by an NSF Graduate Research Fellowship awarded to A.S., National Institutes of Health Director's Pioneer Award (DP1OD003312) to L.F.B., and NIH R01EY019477 and NSF 0842947 to M.B.

\section{References}

Addis, D. R., Wong, A. T., \& Schacter, D. L. (2007). Remembering the past and imagining the future: Common and distinct neural substrates during event construction and elaboration. Neuropsychologia, 45(7), 1363-1377.

Aminoff, E., Schacter, D. L., \& Bar, M. (2008). The cortical underpinnings of context-based memory distortion. Journal of Cognitive Neuroscience, 20(12), 2226-2237.

Andrews-Hanna, J. R., Reidler, J. S., Sepulcre, J., Poulin, R., \& Buckner, R. L. (2010). Functional-anatomic fractionation of the brain's default network. Neuron, 65(4), 550-562.

Baddeley, A. (1972). Selective attention and performance in dangerous environments. British Journal of Psychology, 63, 537-546.

Bar, M. (2004). Visual objects in context. Nature Reviews Neuroscience, $5(8), 617-629$.

Bar, M. (2009). The proactive brain: Memory for predictions. Philosophical Transactions of the Royal Society of London. Series B, Biological Sciences, 364(1521), 1235-1243.

Bar, M., \& Aminoff, E. (2003). Cortical analysis of visual context. Neuron, 38(2), 347-358.

Bar, M., Aminoff, E., Mason, M., \& Fenske, M. (2007). The units of thought. Hippocampus, 17(6), 420-428.

Bar, M., Shenhav, A., \& Devaney, K. (2012). Humans like associative objects. Manuscript submitted for publication

Barbas, H. (2007a). Flow of information for emotions through temporal and orbitofrontal pathways. Journal of Anatomy, 211(2), 237249.

Barbas, H. (2007b). Specialized elements of orbitofrontal cortex in primates. Annals of the New York Academy of Sciences, 1121, $10-32$.

Bargh, J., \& Chartrand, T. (1999). The unbearable automaticity of being. American Psychologist, 54(7), 462-479.

Barrett, L. F. (2006). Valence is a basic building block of emotional life. Journal of Research in Personality, 40(1), 35-55.

Barrett, L. F., \& Bar, M. (2009). See it with feeling: Affective predictions during object perception. Philosophical Transactions of the Royal Society B: Biological Sciences, 364(1521), 1325-1334.
Barrett, L. F., \& Bliss-Moreau, E. (2009). Affect as a psychological primitive. Advances in Experimental Social Psychology, 41, 167-218.

Barsalou, L. W. (2009). Simulation, situated conceptualization, and prediction. Philosophical Transactions of the Royal Society of London. Series B, Biological Sciences, 364(1521), 1281-1289.

Bartlett, F. C. (1932). Remembering. Cambridge: Cambridge University Press.

Bornstein, A. M., Nylen, E. L., \& Steele, S. A. (2011). Unblocking the neural substrates of model-based value. Journal of Neuroscience, 31(28), 10117-10118. Comment.

Bower, G. H. (2008). The evolution of a cognitive psychologist: A journey from simple behaviors to complex mental acts. Annual Review of Psychology, 59, 1-27.

Brainard, D. H. (1997). The psychophysics toolbox. Spatial Vision, 10, 433-436.

Brown, S., Gao, X., Tisdelle, L., Eickhoff, S. B., \& Liotti, M. (2011). Naturalizing aesthetics: Brain areas for aesthetic appraisal across sensory modalities. NeuroImage, 58(1), 250-258.

Brunye, T. T., Gagnon, S. A., Paczynski, M., Shenhav, A., Mahoney, C. R., \& Taylor, H. A. (2012). Happiness by association: Generating broad associations promotes positive affect. Manuscript under review.

Buckner, R. L., Andrews-Hanna, J. R., \& Schacter, D. L. (2008). The brain's default network: Anatomy, function, and relevance to disease. Annals of the New York Academy of Sciences, 1124, 1-38.

Bunce, J. G., \& Barbas, H. (2011). Prefrontal pathways target excitatory and inhibitory systems in memory-related medial temporal cortices. NeuroImage, 55(4), 1461-1474.

Burianova, H., \& Grady, C. L. (2007). Common and unique neural activations in autobiographical, episodic, and semantic retrieval. Journal of Cognitive Neuroscience, 19(9), 1520-1534.

Cabanac, M. (2002). What is emotion? Behavioural Processes, 60(2), 69-83.

Carmichael, S. T., \& Price, J. L. (1995a). Limbic connections of the orbital and medial prefrontal cortex in macaque monkeys. The Journal of Comparative Neurology, 363(4), 615-641.

Carmichael, S. T., \& Price, J. L. (1995b). Sensory and premotor connections of the orbital and medial prefrontal cortex of macaque monkeys. The Journal of Comparative Neurology, 363(4), $642-664$.

Chaumon, M., Kveraga, K., Barrett, L. F., \& Bar, M. (2012). Visual predictions in the orbitofrontal cortex rely on the identifiability of the stimuli. Manuscript under review.

Chib, V. S., Rangel, A., Shimojo, S., \& O'Doherty, J. P. (2009). Evidence for a common representation of decision values for dissimilar goods in human ventromedial prefrontal cortex. Journal of Neuroscience, 29(39), 12315-12320.

Christoff, K., Gordon, A. M., Smallwood, J., Smith, R., \& Schooler, J. W. (2009). Experience sampling during fMRI reveals default network and executive system contributions to mind wandering. Proceedings of the National Academy of Sciences of the United States of America, 106(21), 8719-8724.

Chun, M. M., \& Jiang, Y. (2003). Implicit, long-term spatial contextual memory. Journal of Experimental Psychology: Learning, Memory, and Cognition, 29(2), 224-234.

Clore, G., \& Huntsinger, J. (2007). How emotions inform judgment and regulate thought. Trends in Cognitive Sciences, 11(9), 393399.

D'Argembeau, A., Stawarczyk, D., Majerus, S., Collette, F., Van der Linden, M., Feyers, D., et al. (2009). The neural basis of personal goal processing when envisioning future events. Journal of Cognitive Neuroscience, 22(8), 1701-1713.

Damasio, A. R. (1994). Descartes' error: Emotion, reason, and the human brain. New York: G.P. Putnam.

Damasio, A. R. (1996). The somatic marker hypothesis and the possible functions of the prefrontal cortex. Philosophical Transactions 
of the Royal Society of London. Series B, Biological Sciences, 351 (1346), 1413-1420.

Daw, N. D., O'Doherty, J. P., Dayan, P., Seymour, B., \& Dolan, R. J. (2006). Cortical substrates for exploratory decisions in humans. Nature, 441(7095), 876-879.

Daw, N. D., Gershman, S. J., Seymour, B., Dayan, P., \& Dolan, R. J. (2011). Model-based influences on humans' choices and striatal prediction errors. Neuron, 69, 1204-1215.

Deichmann, R., Gottfried, J. A., Hutton, C., \& Turner, R. (2003). Optimized EPI for fMRI studies of the orbitofrontal cortex. NeuroImage, 19(2 Pt 1), 430-441.

Denkova, E., Botzung, A., Scheiber, C., \& Manning, L. (2006). Implicit emotion during recollection of past events: A nonverbal fMRI study. [Comparative Study]. Brain Research, 1078(1), 143-150.

Desikan, R. S., Ségonne, F., Fischl, B., Quinn, B. T., Dickerson, B. C., Blacker, D., et al. (2006). An automated labeling system for subdividing the human cerebral cortex on MRI scans into gyral based regions of interest. NeuroImage, 31(3), 968-980.

Eichenbaum, H., \& Fortin, N. (2009). The neurobiology of memory based predictions. Philosophical Transactions of the Royal Society of London. Series B, Biological Sciences, 364(1521), 1183-1191

Fischl, B., Salat, D. H., Busa, E., Albert, M., Dieterich, M., Haselgrove, C., et al. (2002). Whole brain segmentation: Automated labeling of neuroanatomical structures in the human brain. Neuron, 33(3), 341-355.

Fischl, B., van der Kouwe, A., Destrieux, C., Halgren, E., Segonne, F., Salat, D., et al. (2004). Automatically parcellating the human cerebral cortex. Cerebral Cortex, 14(1), 11.

Fredrickson, B. L. (2004). The broaden-and-build theory of positive emotions. Philosophical Transactions of the Royal Society of London. Series B, Biological Sciences, 359(1449), 1367-1378.

Gasper, K., \& Clore, G. (2002). Attending to the big picture: Mood and global versus local processing of visual information. Psychological Science, 13(1), 34.

Grabenhorst, F., \& Rolls, E. T. (2008). Selective attention to affective value alters how the brain processes taste stimuli. The European Journal of Neuroscience, 27(3), 723-729.

Grabenhorst, F., \& Rolls, E. T. (2011). Value, pleasure and choice in the ventral prefrontal cortex. Trends in Cognitive Sciences, 15(2), 56-67.

Haber, S. N., \& Knutson, B. (2010). The reward circuit: Linking primate anatomy and human imaging. Neuropsychopharmacology, 35(1), 4-26.

Hampton, A. N., Bossaerts, P., \& O'Doherty, J. P. (2006). The role of the ventromedial prefrontal cortex in abstract state-based inference during decision making in humans. Journal of Neuroscience, 26(32), 8360-8367.

Isen, A. M., Johnson, M. M., Mertz, E., \& Robinson, G. F. (1985). The influence of positive affect on the unusualness of word associations. Journal of Personality and Social Psychology, 48(6), 1413-1426.

James, W. (1890). The principles of psychology. New York: Holt.

Kable, J. W., \& Glimcher, P. W. (2009). The neurobiology of decision: Consensus and controversy. Neuron, 63(6), 733-745.

Kalisch, R., Korenfeld, E., Stephan, K. E., Weiskopf, N., Seymour, B., \& Dolan, R. J. (2006). Context-dependent human extinction memory is mediated by a ventromedial prefrontal and hippocampal network. Journal of Neuroscience, 26(37), 9503-9511.

Kennerley, S. W., Dahmubed, A. F., Lara, A. H., \& Wallis, J. D. (2009). Neurons in the frontal lobe encode the value of multiple decision variables. Journal of Cognitive Neuroscience, 21(6), $1162-1178$

Kim, H., Shimojo, S., \& O'Doherty, J. P. (2006). Is avoiding an aversive outcome rewarding? Neural substrates of avoidance learning in the human brain. PLoS Biology, 4(8), e233.

Kober, H., Barrett, L. F., Joseph, J., Bliss-Moreau, E., Lindquist, K. A., \& Wager, T. D. (2008). Functional grouping and cortical- subcortical interactions in emotion: A meta-analysis of neuroimaging studies. Neuroimage, 42(2), 998-1031.

Kringelbach, M. L., \& Berridge, K. C. (2009). Towards a functional neuroanatomy of pleasure and happiness. Trends in Cognitive Sciences, 13(11), 479-487.

Kumaran, D., Summerfield, J. J., Hassabis, D., \& Maguire, E. A. (2009). Tracking the emergence of conceptual knowledge during human decision making. Neuron, 63(6), 889-901.

Kurth-Nelson, Z., Bickel, W., \& Redish, A. D. (2012). A theoretical account of cognitive effects in delay discounting. The European Journal of Neuroscience, 35(7), 1052-1064.

Kveraga, K., Ghuman, A., \& Bar, M. (2007). Top-down predictions in the cognitive brain. Brain and Cognition, 65(2), 145-168.

Kveraga, K., Ghuman, A. S., Kassam, K. S., Aminoff, E. A., Hämäläinen, M. S., Chaumon, M., et al. (2011). Early onset of neural synchronization in the contextual associations network. Proceedings of the National Academy of Sciences, 108(8), 3389.

Lang, P., Bradley, M., \& Cuthbert, B. (2008). International affective picture system (IAPS): Affective ratings of pictures and instruction manual. Technical Report A-8. Gainesville: University of Florida.

Lebreton, M., Jorge, S., Michel, V., Thirion, B., \& Pessiglione, M. (2009). An automatic valuation system in the human brain: Evidence from functional neuroimaging. Neuron, 64(3), 431-439.

Liu, X., Hairston, J., Schrier, M., \& Fan, J. (2011). Common and distinct networks underlying reward valence and processing stages: A meta-analysis of functional neuroimaging studies. Neuroscience and Biobehavioral Reviews, 35(5), 1219-1236.

Mason, M. F., \& Bar, M. (2011). The effect of mental progression on mood. Journal of Experimental Psychology. General, 141(2), 217-221.

Mason, M. F., Norton, M. I., Van Horn, J. D., Wegner, D. M., Grafton, S. T., \& Macrae, C. N. (2007). Wandering minds: The default network and stimulus-independent thought. Science, 315(5810), 393-395.

Milad, M. R., Wright, C. I., Orr, S. P., Pitman, R. K., Quirk, G. J., \& Rauch, S. L. (2007). Recall of fear extinction in humans activates the ventromedial prefrontal cortex and hippocampus in concert. Biological Psychiatry, 62(5), 446-454.

Murray, E. A., \& Wise, S. P. (2010). Interactions between orbital prefrontal cortex and amygdala: Advanced cognition, learned responses and instinctive behaviors. Current Opinion in Neurobiology, 20(2), 212-220.

Nichols, T., Brett, M., Andersson, J., Wager, T., \& Poline, J.-B. (2005). Valid conjunction inference with the minimum statistic. NeuroImage, 25(3), 653-660.

Nielen, M. M. A., Heslenfeld, D. J., Heinen, K., Van Strien, J. W., Witter, M. P., Jonker, C., et al. (2009). Distinct brain systems underlie the processing of valence and arousal of affective pictures. Brain and Cognition, 71(3), 387-396.

O'Doherty, J. P., Critchley, H., Deichmann, R., \& Dolan, R. J. (2003a). Dissociating valence of outcome from behavioral control in human orbital and ventral prefrontal cortices. Journal of Neuroscience, 23 (21), 7931-7939.

O'Doherty, J. P., Kringelbach, M. L., Rolls, E. T., Hornak, J., \& Andrews, C. (2001). Abstract reward and punishment representations in the human orbitofrontal cortex. Nature Neuroscience, 4 (1), 95-102.

O'Doherty, J. P., Winston, J., Critchley, H. D., Perrett, D., Burt, D. M., \& Dolan, R. J. (2003b). Beauty in a smile: The role of medial orbitofrontal cortex in facial attractiveness. Neuropsychologia, 41 (2), 147-155.

Oliva, A., \& Torralba, A. (2007). The role of context in object recognition. Trends in Cognitive Sciences, 11(12), 520-527.

Öngür, D., \& Price, J. L. (2000). The organization of networks within the orbital and medial prefrontal cortex of rats, monkeys and humans. Cerebral Cortex, 10(3), 206-219.

Padoa-Schioppa, C., \& Assad, J. A. (2006). Neurons in the orbitofrontal cortex encode economic value. Nature, 441(7090), 223-226. 
Padoa-Schioppa, C., \& Cai, X. (2011). The orbitofrontal cortex and the computation of subjective value: Consolidated concepts and new perspectives. Annals of the New York Academy of Sciences, 1239 (1), 130-137.

Peters, J. (2011). The role of the medial orbitofrontal cortex in intertemporal choice: Prospection or valuation? Journal of Neuroscience, 31 (16), 5889-5890.

Peters, J., \& Buchel, C. (2010). Episodic future thinking reduces reward delay discounting through an enhancement of prefrontal-mediotemporal interactions. Neuron, 66(1), 138148.

Rangel, A., Camerer, C., \& Montague, P. R. (2008). A framework for studying the neurobiology of value-based decision making. Nature Reviews Neuroscience, 9(7), 545-556.

Rangel, A., \& Hare, T. A. (2010). Neural computations associated with goal-directed choice. Current Opinion in Neurobiology, 20(2), 262-270.

Ritchey, M., Bessette-Symons, B., Hayes, S. M., \& Cabeza, R. (2011). Emotion processing in the aging brain is modulated by semantic elaboration. Neuropsychologia, 49(4), 640-650.

Rolls, E. T. (1986). Neural systems involved in emotion in primates. In R. Plutchik \& H. Kellerman (Eds.), Emotion: Theory, research, and experience (Biological Foundations of Emotion, Vol. 3, pp. 125-143). New York: Academic Press.

Rolls, E. T., Kringelbach, M. L., \& de Araujo, I. E. T. (2003). Different representations of pleasant and unpleasant odours in the human brain. The European Journal of Neuroscience, 18 (3), 695-703.

Roy, M., Shohamy, D., \& Wager, T. D. (2012). Ventromedial prefrontal-subcortical systems and the generation of affective meaning. Trends in Cognitive Sciences, 16(3), 147-156.

Russell, J. (2003). Core affect and the psychological construction of emotion. Psychological Review, 110(1), 145-172.
Sass, K., Habel, U., Sachs, O., Huber, W., Gauggel, S., \& Kircher, T. (2011). The influence of emotional associations on the neural correlates of semantic priming. Human Brain Mapping, 33(3), 676-694.

Schoenbaum, G., Takahashi, Y., Liu, T.-L., \& Mcdannald, M. A. (2011). Does the orbitofrontal cortex signal value? Annals of the New York Academy of Sciences, 1239(1), 87-99.

Seymour, B., O'Doherty, J. P., Koltzenburg, M., Wiech, K., Frackowiak, R., Friston, K., et al. (2005). Opponent appetitiveaversive neural processes underlie predictive learning of pain relief. Nature Neuroscience, 8(9), 1234-1240.

Spreng, R. N., Mar, R. A., \& Kim, A. S. N. (2009). The common neural basis of autobiographical memory, prospection, navigation, theory of mind, and the default mode: A quantitative metaanalysis. Journal of Cognitive Neuroscience, 21(3), 489-510.

Summerfield, C., Egner, T., Greene, M., Koechlin, E., Mangels, J., \& Hirsch, J. (2006). Predictive codes for forthcoming perception in the frontal cortex. Science, 314(5803), 1311.

Szpunar, K. K., Watson, J. M., \& McDermott, K. B. (2007). Neural substrates of envisioning the future. Proceedings of the National Academy of Sciences of the United States of America, 104(2), 642-647.

Thirion, B., Pinel, P., Mériaux, S., Roche, A., Dehaene, S., \& Poline, J. B. (2007). Analysis of a large fMRI cohort: Statistical and methodological issues for group analyses. NeuroImage, 35(1), 105-120.

Wallis, J. D. (2007). Orbitofrontal cortex and its contribution to decision-making. Annual Review of Neuroscience, 30, 31-56.

Wallis, J. D., \& Kennerley, S. W. (2011). Contrasting reward signals in the orbitofrontal cortex and anterior cingulate cortex. Annals of the New York Academy of Sciences, 1239(1), 33-42.

Weiskrantz, L. (1968). Emotion. In L. Weiskrantz (Ed.), Analysis of behavioural change (pp. 50-90). New York: Harper and Row.

Zajonc, R. (1980). Feeling and thinking: Preferences need no inferences. American Psychologist, 35(2), 151-175. 